\title{
Professional AI Chess Board Autonomous Chess Board (Human Vs. Robot)
}

\author{
Kalpesh H. Zurange \\ Electronics and Telecommunication \\ PESs Modern College Of Engineering, Pune,India
}

\begin{abstract}
Chess - the classic game has come a long way from ancient times to modern days but yet remains a mind boggling challenge of intense strategic play and patience.

Artificial intelligence is booming branch in technology which can be simply defined as "Any device /software that perceives its environment and takes actions that maximize its chance of successfully achieving its goals which is often compared to the 'cognitive' functions that humans associate with human minds, such as 'learning' and 'problem solving'."

The project aims to combine both these entities to create a selfplaying chess board which has the capacity to stand on its own as a playing unit for a single person without any opponent. The pieces on the board will automatically be moved on the board according to AI so as to beat the person in front and thus create an unparalleled experience for a chess player. The strong structure consisting of various parts and sensors along with a fast processing unit.

The introduction of AI brings a completely new dynamic into the game as the machine will improve its game strategies with time opening a completely new prospect in the game.

A motor based system, along with smart magnetic detection provides a low noise and friction product at lower power consumption along with less wear and tear and even more effectively brings the cost of the product down sizably It helps in providing unique features such as hassle free motion of pieces along with unprecedented detection of moves are just some of the features the project aims to cover against the currently available similar products available in today's market.

Also an improved size of the board along with aesthetically pleasing pieces is one of the feature the project aims to complete over its other competitors.
\end{abstract}

Keywords-Human Robot Interaction, Artificial Intelligence, Self-Playing.

\section{INTRODUCTION}

Chess is a two-player strategy board game played on a chessboard, a checkered game board with 64 squares arranged in an $8 \times 8$ grid. The game is played by millions of

People worldwide. Chess is believed to have originated in India sometime before the 7 th century.

Though the game is complex one, it is based on approaching, threatening and capturing pieces until destination is reached. John Artise in Chess and Education states: "Visual stimuli tend to improve memory more than any other stimuli; chess is definitely an excellent memory exerciser the effects of which are transferable to other subjects where memory is necessary."

The classic game has come a long way from ancient times to modern days but yet remains a mind boggling challenge of intense strategic play and patience. Its endless no. of permutations and combinations is what makes it so interesting every time a new game is played

Artificial intelligence is booming branch in technology which can be simply defined as "Any device /software that perceives its environment and takes actions that maximize its chance of successfully achieving its goals which is often compared to the "cognitive" functions that humans associate with human minds, such as "learning" and "problem solving".'

In recent years AI is successfully associated with applications such as understanding human speech, competing at the highest level in strategic game systems (such as chess and Go),autonomously operating cars, and intelligent routing in content delivery networks and military simulations.

The project aims to combine both these entities to create a selfplaying chess board which has the capacity to stand on its own as a playing unit for a single person without any opponent.

The pieces on the board will automatically be moved on the board according to AI to as the device will try to beat the person in front and thus create an unparalleled experience for a chess player.

As the world gets more and more technology oriented use of devices such as cell phones

Laptops etc. is on the rise. This result in excessive screen time which results in irreversible damages to eye site the effect is even worse in children. Thus the project provides a real chess partner instead of a virtual experience

The experience between the virtually available chess and the real chess playing experience is immense and not matched in the virtual world. The project provides a professional chess player with a simple training companion where he can train as long as he /she wants it uses powerful processors such an Arduino mega and Raspberry pi3 along with a Stockfish engine. The Arm motion is carried out by 2 stepper motor with 1200steps/rev as the base joint. Then there are 1 servo motors which are light weight yet effective at the $\mathrm{Z}$ Axis use for pieces Pick and Place.

The reed switches are magnetically switching devices which are used to detect human moves and thus send information about the human moves. Forward and reverse kinematics will be used to easily provide accurate precise and simple motion of the robotic Frame.

Thus the project provide the real chess playing experience using modern technological tools and artificial intelligence. 


\section{METHOD}

\section{THE H-BOT CONCEPT}

This H-Bot concept represents the interaction of

Two rotary drives which are connected by a Single H-shaped circumferential timing belt around two staggered linear axes in a gantry type like configuration, see figure 1 . The kinematics of the H-Bot mechanism formulated in (1), where $\mathrm{x}$ and $\mathrm{y}$ are the TCP- and q1 and q2 are the drive-coordinates in radians is the drive's pulleys radius. Due to the fact that the drives do not have to be moved the achievable dynamic values can be quite high. Another aspect of this concept in contrast to delta-robot like kinematics is that the weight of the work load is carried by the linear guide ways and not by the drives. Also, the working envelope, the required transformations and the uniformity of the distribution of properties are far easier to handle for industrial use than the often odd shaped working envelopes, complex kinematics and the strongly nonlinear property distribution of alternative concepts

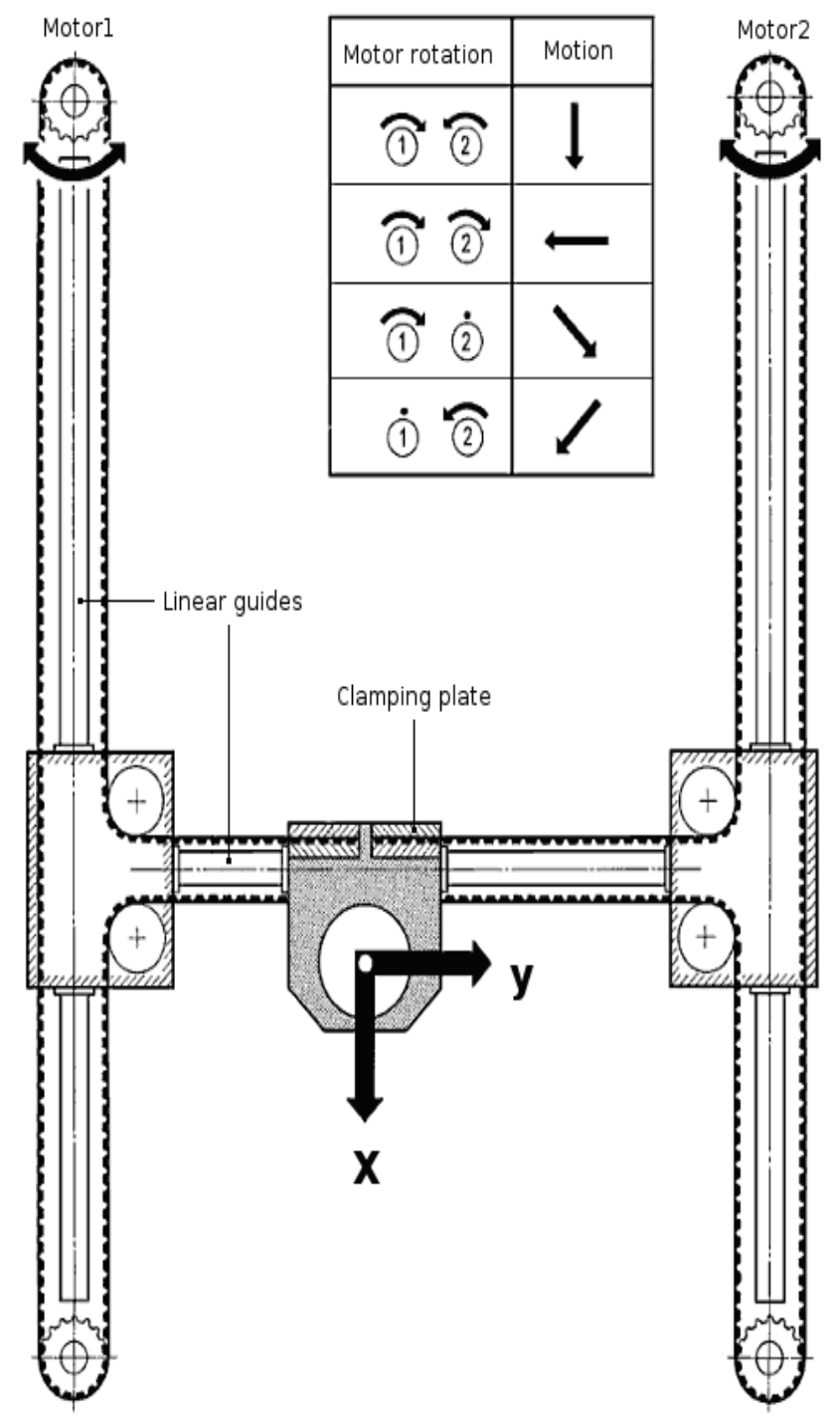

FIGURE 1. Schematic of the H-Bot configuration and the kinematic [3], numbering of bodies

\section{The reed switch matrix}

64 reed switches are needed to build a $8 \times 8$ matrix. There's a big difference with a micro switch matrix as used. Any square containing a piece will have its reed switch activated. In a matrix like this can occur "ghost" activations, i.e., when the microcontroller scans the matrix it "can think" that there are some switches activated when in fact they are not. This effect, known as "ghost key", is solved by adding a diode in series with each of the switches.

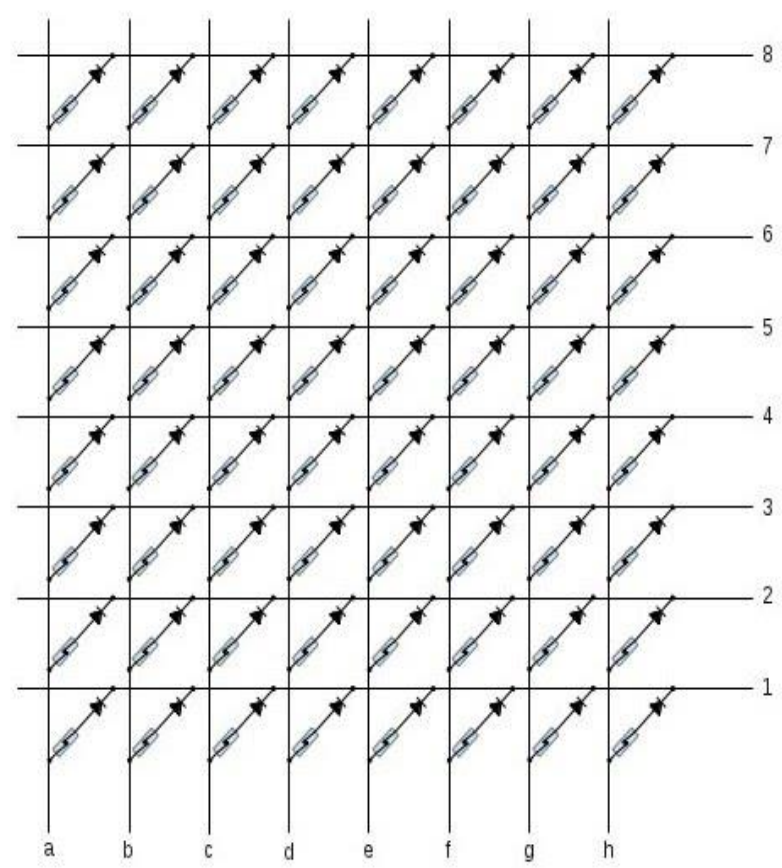

III. MEASUREMENT RESULTS

On a first prototype a series of measurements covering geometric properties could be carried out.

Figure 2 shows exemplarily the results of a circle test. As opposed to measurements on serial axis concepts the systematic deviations show additional systematic effects: At axis reversal similar effects are noticed as are typical for axis having semi-closed position feed-back. In addition to these at the axes reversals similar affects are visible at positions on the $\pm 45^{\circ}$ diagonals. These effects are caused by drive reversal, where station occurs on the driven pulley and/or motor bearing in combination with backlash of the belt. Based on these first measurements on the prototype, a number of improvements could be achieved using feed forward torque computation. By this effects of friction and inertia could strongly be reduced as can be seen in Figure 3.

Especially the effects on the \pm 45 locations could be reduced significantly. For pick and place applications that the robot is designed for, position accuracy is only important at the end of a motion. In Figure 4 the results of a positioning measurement according to ISO 230-2 are shown. As can be seen, a quite good repeatability is obtained, when regarding unidirectional approach of measuring positions. The reversals are quite large and slightly position dependent. The position deviations show 
a systematic slope, which could be reduced, when applying linear pitch error compensation.

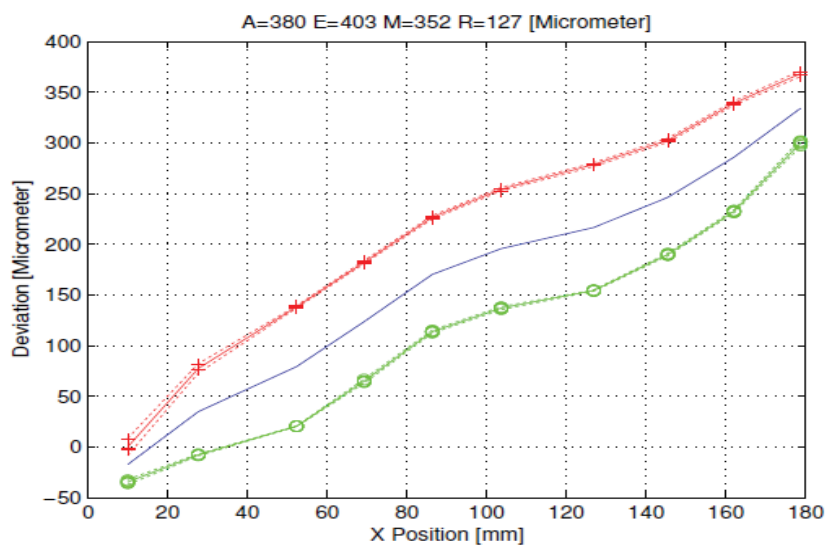

FIGURE 2. Positioning results in X-direction on the H-Bot prototype.

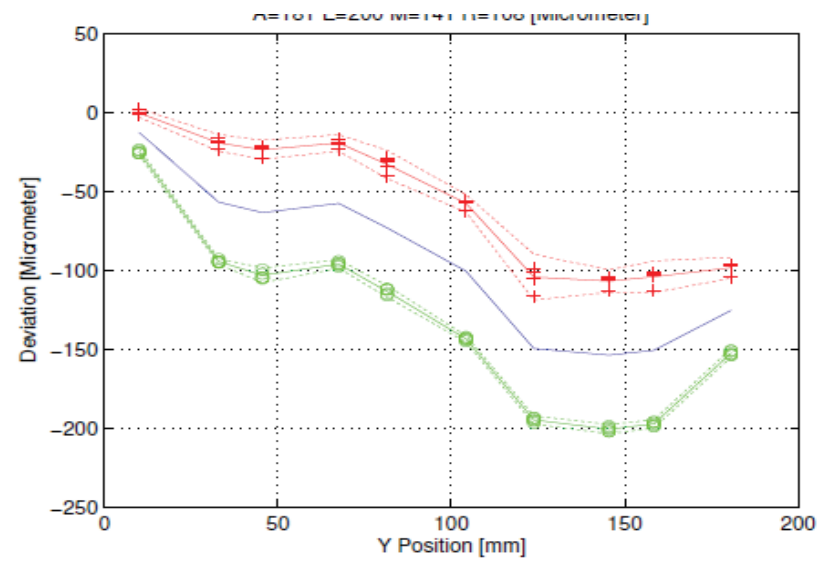

FIGURE 3. Positioning results in Y-direction on the H-Bot prototype.

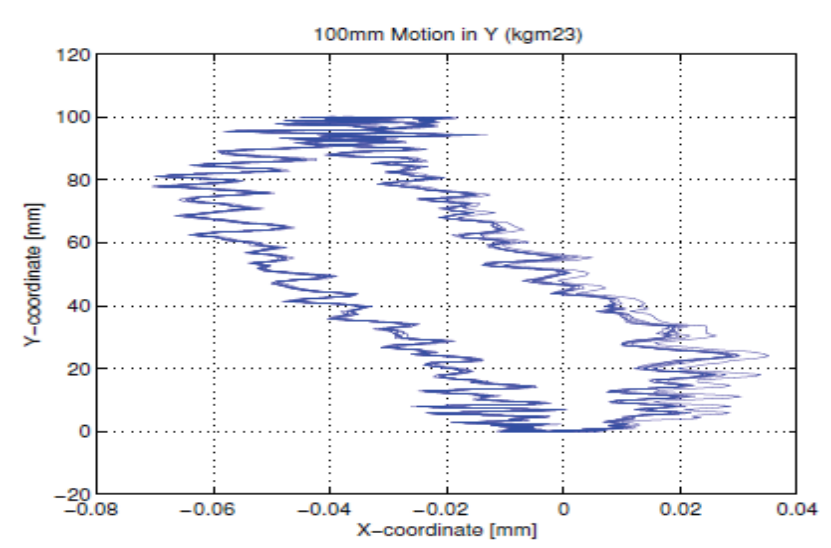

FIGURE 4. 2D-Path measurements (cross-grid) for a series of pendulum movements over $100 \mathrm{~mm}$ in $\mathrm{Y}$-direction at $0.4 \mathrm{~m} / \mathrm{sec}$ feed rate.

\section{FLOW CHART}

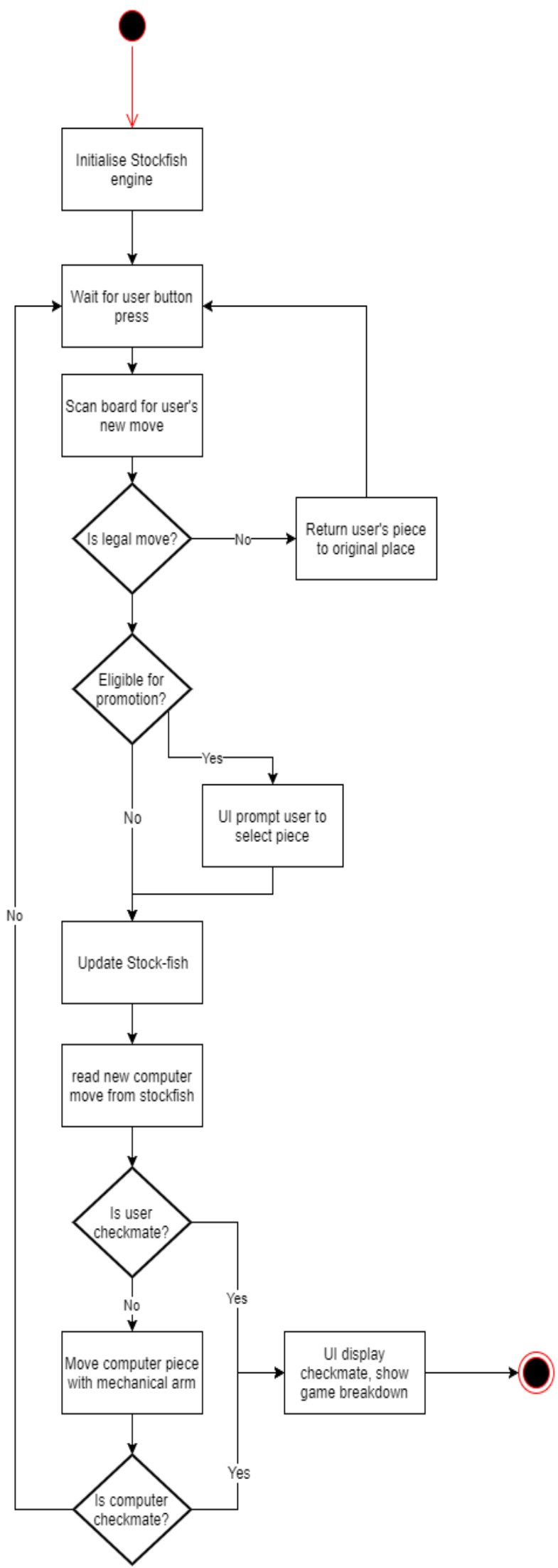




\section{RESULTS}

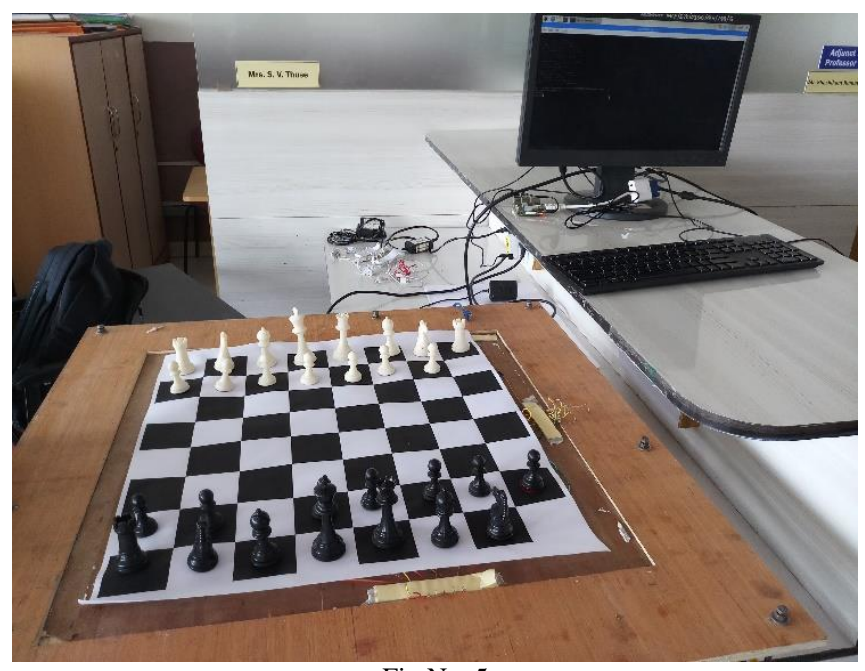

Fig No. 5

- A working model of the Chess Board

- To make it easier for players not only to play the game of chess but also to learn new techniques

- The game shouldn't be player centric, it should be as much applicable to a beginner as to a professional

- The movement of the pieces should be natural and not mechanical

- The player should have a sense of playing a human and not a machine

\section{DISCUSSION AND CONCLUSION}

A. The Advancements in technology haven't entered the game as of now . The physical chess playing experience is not matched in the digital world

$B$. Thus the project will help to bridge this gap and provide a hassle free experience for a human to play against a AI based system

C. It's time to move back from a virtual world to the real world. And not just moving back but taking the best out of both the worlds. In this project me make use of AI to help our mind be better at critical thinking. It is supposed to serve as a tool to the mind.

\section{ACKNOWALDEGMENT}

We thank Prof. Dr. Mrs. R. Kamathe ma'am (HOD) for allowing us to use the various components available and other things such as microcontrollers necessary for the completion of our project.

We thank our project guide Mrs. Aparna More for giving us a platform to show and develop our project skills.

We thank her also for helping us in developing and implementing the entire project and to improve our technical skills. We got time to time feedback from her which helped us to develop our Project.

\section{REFRENCES}

[1] Untold History of AI: When Charles Babbage Played Chess With the Original Mechanical Turk, spectrum.ieee.org

[2] www.stockfishengin.com

[3] www.squareoff.com

[4] James Zhang "Autonoumans Chess Board Development"

[5] Bhavya Goil Attur Mehta https://www.kickstarter.com/projects/infivention/square-off-worldssmartest-chess-board-relaunched/description

[6] Cynthia Matuszek et all, "Gambit: An Autonomous Chess-Playing Robotic System," 2011 IEEE International Conference on Robotics and Automation.

[7] David B. Fogel, Timothy J. Hays, Sarah L. Hahn, James Quon, , "The Blondie25 Chess Program Competes Against Fritz 8.0 and a Human Chess Master", 2006 IEEE.

[8] Hans Berliner, "Hitech Chess: From Master toSenior Master with no Hardware Change", International Workshop on Industrial Application of Machine Intelligence and Vision (MIV89), Tokyo, April 1012,1989 .

[9] De la Escalera and J. M. Armingol, "Automatic chessboard detection for intrinsic and extrinsic camera parameter calibration," Sensors, vol. 10, no. 3, pp. 2027-2044, 2010.

[10] Akash

deep _deep

[11] T. COUR, R. LAURANSON, and M. VACHETTE, "Autonomous chessplaying robot," Ecole Polytechnique, July, 2002.

[12] C. Matuszek, B. Mayton, R. Aimi, M. P. Deisenroth, L. Bo, and D. Fox, "Gambit: A robust chess-playing robotic system," in Proc. of the IEEE International Conference on Robotics and Automation, 2011. 